\title{
ARCHITECTURE DES COLLÈGES ET COMMUNICATION DANS TROIS CONSEILS GÉNÉRAUX EN FRANCE (1986-2001)
}

\author{
Florent Champy ${ }^{1}$
}

L'utilisation de l'architecture à des fins de communication, qui a été une constante du comportement des Princes, est encore fréquente dans les ministères et les administrations ${ }^{2}$. Après les lois de décentralisation promulguées en France entre 1982 et 1986, ce phénomène a vite touché les collectivités territoriales, dotées de compétences et de moyens nouveaux. Les grandes villes en compétition pour le statut de métropoles européennes se sont lancées dans des opérations qui devaient rehausser leur image, en dépensant parfois des sommes importantes et en recourant aux services d'architectes célèbres ${ }^{3}$. Des assemblées régionales ou départementales ont ainsi décidé la construction d'Hôtels de région ou de département qui ont pu être qualifiés de pharaoniques. A travers ces réalisations, les collectivités

1 Chargé de recherche au CNRS. Centre de Sociologie des Arts, EHESS, Paris.

2 Fl. CHAMPY, Les architectes et la commande publique, Paris, PUF, coll. "Sociologie", 1998.

3 V. BIAU, L'architecture comme emblème municipal, Paris, Plan construction, coll. "Recherches", 1991 ; Fr. CHASLIN, "L'architecture à l'heure du marketing municipal", Raison présente, n 102, 1992, pp. 13-20; Fl. CHAMPY, Sociologie de l'architecture, Paris, La Découverte, coll. "Repères", 2001.

Recherches en communication, $\mathrm{n}^{\circ} 18$ (2002). 
territoriales ont voulu donner une image de modernité, de pouvoir et de dynamisme, ce qui devait aider à faire venir des activités nouvelles et des habitants des classes moyennes et supérieures.

La forte dimension symbolique des réalisations ne doit cependant pas faire perdre de vue que l'architecture est aussi - et surtout- affaire de fonction. Aucun bâtiment n'en est dénué. Même quand la volonté de communiquer s'est révélée déterminante dès le lancement des projets, comme ce fut le cas pour les grands travaux mitterrandiens ${ }^{1}$, il a bien fallu trouver à chaque construction sa fonction, son contenu et ses occupants. L'Opéra Bastille, symbole d'un rééquilibrage du centre de gravité culturel de Paris vers l'Est inauguré le 14 juillet 1989, est aussi la traduction d'un projet de démocratisation de l'art lyrique, consistant à rendre l'opéra accessible à un large public populaire par la constitution d'un répertoire, par opposition à l'élitisme du Palais Garnier, grâce à une salle de 2700 places et à un dispositif scénique qui permet les changements rapides de décors et donc l'alternance de spectacles différents ${ }^{2}$. Projet culturel, architecture, constitution du programme lyrique et usage du thème de l'opéra populaire dans la communication sont ainsi fortement intriqués. La Très Grande Bibliothèque n'aurait pu voir le jour sans l'annonce de la construction d'une bibliothèque ultramoderne, en réseau, qui a donné son sens au projet, en dépit des critiques ${ }^{3}$ : on voit dans ces deux cas ce que la communication des édifices les plus coûteux, dont la construction fut décidée au sommet de l'Etat, doit à la fonction qu'ils auront à remplir. L'usage de l'architecture à des fins de communication s'articule ainsi avec les choix fonctionnels d'une façon qui peut être fidèle, comme pour l'Opéra Bastille ou au contraire plutôt trompeuse, comme l'illustre la Bibliothèque François-Mitterrand. Ce constat vaut aussi pour les réalisations des collectivités territoriales. Plus proches de leurs électeurs que l'État (notamment quand il s'agit de communes ou de petits départements) les élus locaux sont même souvent plus que lui soucieux de montrer que l'argent du contribuable est dépensé à bon escient, et donc que les bâtiments construits rendent un service à leurs administrés-électeurs.

I Ph. Urfalino, "Décisions, actions et jeux : le cas des grands travaux parisiens", Villes en parallèle, $\mathrm{n}^{\circ} 20-21,1994$, pp. 3-26.

$2 \mathrm{Ph}$. URfalino, Quatre voix pour un opéra: une histoire de l'Opéra Bastille, Paris, Éd. Métailié, 1990.

3 Mandosio, 1999. 
Aussi est-il nécessaire, pour comprendre la politique de communication dont un édifice est le support, de prendre en compte les finalités de ce dernier, c'est-à-dire la définition de son public et de son activité, ainsi que la façon dont ces caractéristiques de l'opération sont présentées dans la communication. Plusieurs questions se posent alors. L'usage de l'architecture à des fins de communication constitue-t-il un objectif essentiel, et donné d'emblée, de la politique menée, ou à l'inverse une activité secondaire, qui peut apparaître tardivement dans le processus de conception et de construction ? La volonté de communiquer pèse-t-elle sur la conduite et le contenu de l'opération, en modifiant les réponses apportées aux problèmes fonctionnels rencontrés, voire sur la décision de la lancer? Notamment, favorise-t-elle les innovations fonctionnelles ? Ou la communication se construit-elle à l'inverse à partir de caractéristiques déterminées par d'autres considérations et qui interviennent comme des données ? Et dans ce cas, quelles propriétés de l'opération en favorisent l'usage dans la communication ? Répondre à ces questions permet seul de saisir les enjeux de la communication autour d'une opération.

Cela ne permet en revanche pas d'épuiser la question de la construction de la communication, car celle-ci se comprend rarement en regardant une opération isolée d'un maître d'ouvrage qui en conduit plusieurs. Il faut en outre se demander comment l'usage de l'architecture à des fins de communication évolue au fur et à mesure que la politique du maître d'ouvrage se déploie. L'activité de communication prend-elle la même forme pour les opérations successives conduites par le même maître d'ouvrage? Quel rôle les opérations passées jouent-elles dans son élaboration? Comment la temporalité de l'activité édilitaire et les traits successifs des opérations menées aident-ils à rendre compte de la construction de la communication?

Ces questions seront abordées ici à partir du cas des constructions de collèges par les conseils généraux. Le choix de cet exemple présente plusieurs avantages. Parce que tous les conseils généraux en France sont concernés par ce type de construction, leur étude offre des possibilités intéressantes de comparaison entre différentes politiques. L'importance des budgets consacrés à ces opérations en fait des enjeux forts pour les élus, ce qui les incite à les valoriser par la communication. La fonction des opérations contribue à leur visibilité, puisque la quasi-totalité des enfants du département, et donc 
indirectement des familles, les fréquentent. Peu d'opérations permettent donc de toucher autant d'électeurs. Enfin, le caractère récent de la décentralisation permet encore d'étudier les politiques de construction de collèges dans toute leur profondeur historique, depuis la dévolution des nouvelles compétences en la matière aux collectivités territoriales en 1986, jusqu'à aujourd'hui' ${ }^{1}$.

Les développements qui suivent s'appuient sur une étude menée dans trois départements : le Nord, les Bouches-du-Rhône et l'Ille-etVilaine. Leur comparaison va permettre d'illustrer les conséquences des stratégies de conduite de la politique édilitaire sur la façon dont la communication s'articule avec la définition fonctionnelle des projets. Mais avant d'exposer les différences entre les politiques menées, il est nécessaire de présenter le cadre commun de lancement de ces politiques, qui est celui de la décentralisation.

\section{La décentralisation et les premières constructions}

Les compétences en matière de construction de collèges sont transmises de l'État aux conseils généraux par une loi de décentrali-

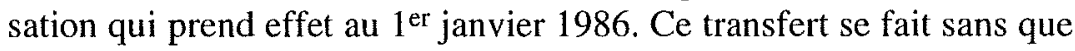
les collectivités territoriales concernées n'aient eu le temps et les moyens de s'y préparer, et de nombreux conseils généraux héritent d'une situation critique qui les oblige à parer au plus pressé. Les bâtiments laissés par l'État sont en effet le plus souvent vétustes et inconfortables, parfois dangereux. Les bâtiments préfabriqués et les constructions métalliques se sont multipliés dans une période où la croissance rapide des effectifs scolarisés obligeait à augmenter dans l'urgence la place disponible. Si la stagnation des effectifs diminue la pression globale, les mouvements de population et l'état du parc de collèges font que des constructions sont encore nécessaires dans bien des départements pour adapter l'offre d'enseignement aux besoins. Dans ces conditions, les choix effectués par les conseils généraux répondent avant tout à des exigences techniques et démographiques.

Les interventions légères, visant à répondre au moindre coût aux problèmes posés, dominent encore jusqu'au début des années 1990.

1 Les développements qui suivent s'appuieront sur les résultats d'une recherche sur La commande publique locale d'architecture, menée dans le cadre d'un contrat avec le ministère de la Culture et de la Communication français, Direction de l'architecture et du patrimoine, entre 1999 et 2001. 
Les réhabilitations sont ainsi partout plus fréquentes que les constructions neuves. De plus, inexpérimentés et ne bénéficiant d'aucune préparation, les conseils généraux s'appuient sur les documents élaborés par l'État, afin de faire l'économie d'études coûteuses et complexes pour lesquelles ils ne disposent ni du temps, ni du personnel qualifié nécessaires. La définition des besoins reste ainsi conforme aux documents types élaborés par l'État et qui servaient à toutes les opérations, dans tous les départements : le nombre d'élèves prévus permet de déduire mécaniquement le nombre de pièces à construire, leur destination et leurs surfaces respectives, selon les grilles étatiques déjà éprouvées.

La reproduction d'un modèle standardisé de collèges ne se prête pas à la valorisation de l'action édilitaire à travers une politique de communication. Il manque encore chez les élus et dans leurs administrations les idées de ce que pourrait en être le message, c'est-à-dire de ce qui pourrait être présenté comme spécifique de leur action, les marges de manœuvre financières permettant de faire des opérations plus "généreuses" que celles de l'État, et un volume de constructions neuves qui favorise la visibilité de cette activité du conseil général.

Ces conditions vont petit à petit commencer à être remplies pour de nombreux conseils généraux, au fur et à mesure que leur activité de construction augmente, et qu'ils peuvent s'appuyer sur l'expérience accumulée au fil des opérations. Un fait est ainsi déterminant pour expliquer la mise en place de politiques de communication au début des années 1990 : la part de plus en plus importante tenue dans les interventions des conseils généraux par les bâtiments neufs, plus propices que les réhabilitations à servir de supports à une politique de communication.

Une date joue un rôle clé dans l'évolution des attitudes des conseils généraux, parce qu'elle correspond à une brutale accélération des constructions dans toute la France. En 1993, à la suite d'un accident survenu à Furiani, les commissions de sécurité qui donnent les autorisations de fonctionnement des collèges multiplient les refus ${ }^{1}$. Pour aider les conseils généraux à faire face à cette nouvelle difficulté, le gouvernement propose des prêts à des taux avantageux pour les opérations lancées dans un délai bref. La conséquence en est

1 Les tribunes d'un stade de football s'écroulent en plein match, faisant de nombreuses victimes parmi les spectateurs. La mise en examen de responsables de la sécurité crée un choc et incite les professionnels à une rigueur nouvelle qui s'accommode mal de l'état de vétusté de certaines constructions. 
l'intensification de l'activité de construction de collèges, ce qui incite à la mise en place d'une politique plus ambitieuse que celle des années antérieures. Dans ce nouveau contexte, tous les conseils généraux ne réagissent pas de la même façon. 1993 constitue ainsi une date clé dans l'individualisation des politiques. La comparaison des politiques menées dans le Nord, les Bouches-du-Rhône et l'Ille-etVilaine permet d'illustrer ce processus de différenciation et de montrer la place qu'y tient la communication.

\section{L'individualisation des politiques}

\section{Diversité des politiques menées et communication}

Le conseil général des Bouches-du-Rhône a mis la communication au centre de sa démarche de construction de collèges, au point qu'elle a fortement influé sur les modalités même de conception des collèges et les caractéristiques des réalisations, tandis que les deux autres utilisent les constructions dans leur communication, mais sans que cette dernière tienne une place très importante et surtout sans qu'elle semble influer sur les choix en matière d'architecture. Comment rendre compte de ces différences ?

La taille du département d'Ille-et-Vilaine peut contribuer à expliquer le peu d'ambition de sa politique de communication. Ce constat est sans surprise, puisque l'étude de politiques architecturales d'administrations avait déjà montré qu'un volume assez important d'activité était une condition nécessaire pour que ces administrations fassent de la construction de leurs équipements un enjeu de communication, indépendamment de la satisfaction des exigences fonctionnelles ${ }^{1}$. Philippe Urfalino a de même montré que dans le cas des grands travaux, le lancement simultané de plusieurs opérations avait seul permis de leur donner cette identité de grands travaux ${ }^{2}$. De façon générale, le volume de construction est un facteur important de valorisation de l'activité édilitaire, notamment dans les médias.

Ainsi, en Ille-et-Vilaine, où la construction de collèges neufs est rare (il s'en est construit quatre en quinze ans), la décision de localisation, le concours, la pose de la première pierre, l'inauguration et la

1 Fl. Champy, Les architectes et la commande publique, op. cit.

${ }^{2} \mathrm{Ph}$. URfalino, "Décisions, actions et jeux...",op. cit. 
première rentrée scolaire donnent bien entendu lieu à communication, au coup par coup (dans la plaquette départementale, par l'édition d'un dossier de presse...) mais il n'y a pas de stratégie forte et continue de valorisation de l'ensemble de la politique de construction du conseil général. L'essentiel de la communication sur la politique d'enseignement de ce dernier porte sur des actions d'une toute autre nature, celles qui permettent de diminuer le coût pour les parents de la scolarité des élèves et d'augmenter le confort de travail des enfants, notamment les plus défavorisés : subventions aux cantines scolaires, achat de livres mis à disposition des établissements et mise en place d'un réseau de ramassage scolaire performant, au profit des villages les plus reculés de ce département encore très rural. Ces actions permettent de donner du département une image sociale, et de toucher l'ensemble des familles d'élèves.

La taille du conseil général et l'importance de son activité de construction ne sauraient cependant tout expliquer. Dans le Nord et les Bouches-du-Rhône, où plusieurs dizaines de collèges ont été construits ou réhabilités depuis la mise en place de la décentralisation, les conseils généraux ont profité du volume de leur activité pour élaborer des politiques originales de construction de collèges qui, au delà de chaque opération prise isolément, forment un tout. Mais ces politiques sont très différentes, notamment si on les regarde sous l'angle de leur rapport à la communication.

Dans les Bouches-du-Rhône, cette dernière figure au premier plan de la politique, au point d'avoir fortement influé sur certains choix. En revanche, si le conseil général du Nord utilise l'architecture dans sa communication, la première ne conditionne cependant jamais la deuxième, elle s'y ajoute. L'essentiel des efforts portent en effet sur des améliorations de la qualité architecturale qui se prêtent mal à valorisation médiatique : des réflexions très techniques sur des thèmes comme la maintenance, la qualité phonique, la couleur ou l'organisation des espaces ont été synthétisées dans des chartes et des performanciels destinés aux professionnels et ignorés du grand public. Ce travail très technique est fait de petites améliorations successives de la connaissance technique des modes de conception des bâtiments, compilées dans des documents arides, dont les effets sur les constructions seront ensuite très diffus, tant dans le temps que dans l'espace. Une des conditions de ces réflexions concerne en effet la temporalité du travail : l'apprentissage ne peut se faire que par incrémentation, en 
constituant peu à peu un corpus de références et d'enseignements à prendre en compte dans les nouvelles opérations.

En conséquence, cette modalité d'apprentissage semble incompatible avec une démarche de valorisation des opérations qui obligerait à figer dès le début du travail les caractéristiques remarquables des opérations, c'est-à-dire celles sur lesquelles la communication s'appuiera. Or le conseil général de Marseille a mis en place dès 1993 une politique de l'architecture où étaient déjà clairement définis et publiquement annoncés certains traits précis des 25 opérations à mener au cours des années suivantes. C'est pourquoi sa politique est particulièrement intéressante pour aider à comprendre comment la communication s'articule avec les autres composantes de la politique architecturale.

\section{La communication au cour de la définition de la politique}

Six traits de cette politique contribuent en effet dès 1993 à sa visibilité et favorisent sa valorisation à travers une politique de communication très importante.

1. Le lancement d'une vague importante de construction s'est fait par l'annonce simultanée de la mise en chantier des études concernant 25 collèges. Cette annonce groupée a permis de donner à cette politique une visibilité tout à fait exceptionnelle : elle a fait de la rénovation du parc de collèges, pourtant à l'œuvre aussi dans les autres départements, un événement singulier, fortement remarqué dans le monde de l'architecture du département, voire de la région. La vertu publicitaire du regroupement des opérations sous un label unique n'est pas sans rappeler les "grands travaux" mitterrandiens, dont une des raisons de leur identification comme tel avait été leur annonce groupée lors d'une conférence de presse présidentielle ${ }^{1}$.

2. Le premier acte de cette nouvelle vague de constructions de collèges a été l'organisation d'une large concertation, pour chacun des 25 collèges concernés, à laquelle participaient les personnels du collège, des élus, des riverains, l'inspection académique, des représentants des associations de parents d'élèves et des représentants des élèves. Cette large concertation contribue à donner au programme de construction son identité et sa visibilité.

1 Ibid. 
3. L'ensemble des opérations a reçu le nom de programme Concerto. Par une dénomination commune, le conseil général a encore accentué la visibilité de l'opération en signifiant clairement qu'il s'agissait d'une démarche d'ensemble ayant une identité propre.

4. Pour conduire ce programme, une petite cellule a été créée, nommée elle aussi Concerto. Autonome tant par rapport à la Direction de l'architecture qu'à la Direction de l'enseignement, elle symbolise la volonté de doter le programme Concerto des moyens administratifs nécessaires à la défense de son identité, loin des routines que ces directions pourraient symboliser. Par ce trait, Concerto s'apparente une fois encore aux grands projets mitterrandiens : un des moyens trouvés pour faire aboutir vite ces opérations extrêmement complexes avait été de créer des administrations de mission, structures légères, dynamiques et supposées plus capables que les services des administrations centrales de promouvoir des innovations ${ }^{1}$ (Urfalino, 1994, Champy, 1999).

5. Des moyens financiers importants ont été consacrés à cette politique, avec trois effets sur les choix effectués. Ils ont permis d'inclure des éléments de programme inhabituels, notamment un amphithéâtre équipé comme salle de spectacle. De plus, confronté à l'urgence de la mise en conformité aux normes de sécurité d'une part importante de son parc de collège, le conseil général a opté pour la reconstruction de la plupart d'entre eux, augmentant ainsi le coût, alors que de nombreux autres conseils généraux avaient plus souvent recours à des réhabilitations. Enfin, la surface des salles de classe a été significativement augmentée par rapport aux conventions de l'Éducation nationale encore utilisées dans d'autres départements.

6. Pour finir, il faut noter l'audace des choix architecturaux effectués : le conseil général a fait appel, pour construire les collèges, à des architectes parfois controversés, en leur laissant une grande liberté d'écriture architecturale. Certaines réalisations ont ainsi suscité des polémiques dans le monde de l'architecture et dans la presse locale.

Les occasions de vanter les mérites de Concerto ont été nombreuses, depuis son lancement : des plaquettes réalisées par le conseil général, une exposition qui se tient dans un théâtre, trois films

1 Ibid.; Fl. CHAMPY, "Comment conduire un grand projet ? Bibliothèque FrançoisMitterrand, Stade de France, Hôpital Georges-Pompidou...", Les Cahiers de la Recherche architecturale et urbaine, $\mathrm{n}^{\circ} 1,1999, \mathrm{pp} .87-100$. 
vidéos, de nombreux articles dans la presse locale. L'examen de ces multiples supports de communication fait ressortir quelques thèmes privilégiés, qui corroborent ce qui précède sur l'originalité du programme. On peut aisément les regrouper en trois catégories.

La première concerne la procédure elle-même de travail. En mettant l'accent sur la large concertation qui a été organisée, le conseil général tente de faire la preuve d'une part de l'intérêt qu'il porte à l'avis de ses administrés, d'autre part de sa capacité à innover tant démocratiquement, avec la participation des riverains, que pédagogiquement, grâce aux contributions des enseignants à l'élaboration du programme. Le recours aux services de jeunes architectes pour conduire ces concertations permet aussi de mettre en avant les efforts du conseil général en faveur des jeunes diplômés, la confiance qu'il leur porte, la modernité et l'audace dont il sait faire preuve. Il faut cependant noter ici que les concertations ont surtout servi à décider quels collèges devraient être reconstruits et quels collèges devraient être réhabilités, sur la base du diagnostic technique effectué par le jeune architecte et qu'elles n'ont en revanche pas influé sur le programme de l'opération. La consultation des usagers s'est révélée avant tout formelle ; elle constituait sa propre fin : il était plus important de pouvoir dire qu'il y avait eu concertation que de modifier effectivement par la concertation le programme des opérations ${ }^{1}$. Sur le fait que concertation visible et efficace ne vont pas toujours de pair.

Le deuxième thème concerne le volontarisme et la générosité de la politique menée, avec la mise en avant des moyens considérables qui ont été débloqués pour les collèges. L'insistance sur le lancement simultané des 25 opérations participe bien entendu de ce thème. De même, la communication du conseil général insiste sur l'augmentation des surfaces consacrées aux élèves, notamment dans les salles de classe. Enfin, le troisième thème concerne les fonctions nouvelles présentes dans les collèges et l'ouverture de ces derniers sur la ville, salles de spectacle et salles informatiques étant mises à la disposition

1 Les raisons pour lesquelles la concertation ne pouvait pas produire de réflexions nouvelles ont été présentées par ailleurs (Fl. CHAMPY, La commande publique locale d'architecture, Rapport pour le ministère de la Culture et de la Communication, Direction de l'architecture et du patrimoine, Paris, DAPA et CSA, nov. 2001 (document non publié). Signalons seulement ici que le caractère standardisé des comptes rendus des jeunes architectes empêchait l'insertion de réflexions sur des thèmes non prévus par l'organisateur de la concertation et que la conduite de la programmation en aval ne permettait pas de prendre en compte les résultats des concertations. 
des habitants du quartier, à la demande du conseil général. Les locaux qui les abritent doivent ainsi être regroupés à l'entrée du collège pour pouvoir être mis à la disposition d'associations aux heures de fermeture de l'établissement. Ces aspects du programme sont ceux qui ont bénéficié du plus de soin dans le travail.

L'étude de la communication menée autour de Concerto permet donc de mettre en évidence les relations ambivalentes qu'elle entretient avec la définition fonctionnelle des projets et l'organisation du travail : elle s'appuie sur ces dernières, tout en imposant sa propre temporalité du travail et en figeant certains choix par les effets d'annonce qu'elle suppose, au détriment de la réflexion fonctionnelle et de l'apprentissage, tel qu'il a peu être mené dans le Nord. Pour saisir les raisons de ces différences, il reste à présenter les conditions de la mise en place de cette politique de communication.

\section{La mise en place d'une politique de communication}

Comment rendre compte du lancement d'une politique qui se prête à une communication aussi construite et maîtrisée ? Il faut mentionner ici trois faits. Cette politique de construction de collèges a été lancée peu de temps après la construction d'un Hôtel du Département audacieux et controversé dans un vieux quartier de Marseille'. Par le souci d'architecture qui est affiché et par la volonté de permettre à des architectes de s'exprimer, elle prolonge cette première grande initiative architecturale du département. Mais elle permet aussi de corriger ce qui a fait l'objet de critiques : le fait d'avoir utilisé l'argent public pour construire un bâtiment qui profite en priorité aux élus et a été conçu par un architecte étranger. Les bénéficiaires sont maintenant de façon incontestable les adolescents du département, ainsi que leurs enseignants. Grâce à la multiplicité des commandes, il est désormais possible de donner du travail à un grand nombre d'architectes locaux. L'audace dont le conseil général fait preuve a donc un précédent : l'usage de l'architecture à des fins de communication a déjà sa place dans ce département.

De plus, la collectivité départementale subit l'émulation du conseil régional, qui a lancé peu de temps auparavant une politique de

1 Il s'agit d'un bâtiment de verre et d'acier dô à l'architecte anglais Aslop, construit dans le vieux quartier Saint-Just. 
construction de lycées déjà servie par une importante communication, le programme «lycées $2000 »$. La concurrence entre les deux collectivités territoriales est renforcée par le fait qu'elles sont de couleurs politiques opposées et que leurs présidents respectifs sont tous deux candidats potentiels aux élections municipales de 1995 à Marseille.

Enfin, cette politique doit beaucoup à la présence parmi les hommes qui ont la confiance du président du conseil général, d'un militant socialiste, architecte de profession. C'est cet homme, déjà très présent lors du concours pour l'Hôtel du département, qui trouve en quelques jours quel parti peut être tiré de l'incitation gouvernementale à lancer vite un grand nombre d'opérations. C'est en effet lui qui a l'idée des concertations simultanées et du recours aux services de jeunes architectes pour les conduire, tout comme il contribue ensuite au choix de concepteurs qui bénéficient déjà d'une bonne notoriété régionale voire nationale. Lors d'une délibération du 16 avril 1993, le contenu des opérations, la façon de les mener, sont encore indéterminés. Dans la délibération du 11 juin 1993, tout est déjà décidé, grâce aux idées que cet architecte a lui-même apportées. Le facteur humain, avec tout ce qu'il peut avoir de contingent, apparaît donc ici comme essentiel.

\section{Conclusion}

La comparaison des politiques de construction de collèges de trois conseils généraux montre qu'il faut éviter de présenter comme naturel un lien entre architecture et communication qui est très variable, se construit dans le temps et dont l'émergence elle-même doit être expliquée. Les relations entre architecture et communication sont ambivalentes : la seconde n'étant pas la fonction principale de la première, elle doit tenir compte des autres dimensions de la politique de construction et notamment des fonctions du bâtiment. Mais elle peut aussi les modifier. Pour ces raisons, l'étude isolée de la communication risque de produire un biais méthodologique préjudiciable à la compréhension même de cet objet de recherche. De même faut-il éviter d'étudier la communication, à un moment donné, sans prendre en compte l'histoire de l'administration ou de la collectivité territoriale concernée. Cette histoire seule permet en effet d'expliquer les compétences et les références qui peuvent être mobilisées dans la construction de la politique. 
Quand la communication se développe, elle est susceptible de se nourrir de thèmes diversifiés. En ne considérant qu'un conseil général, on voit ainsi apparaître dans sa communication l'audace et la modernité des formes architecturales, la procédure suivie, avec les larges concertations qui expriment le souci de l'usager et la rigueur démocratique, les nouveaux services offerts aux collégiens et aux habitants du quartier. Les caractéristiques de l'opération influent bien entendu sur les possibilités de la mettre en valeur par la communication. Mais symétriquement, cette dernière pèse sur les choix architecturaux: elle pousse à l'innovation (qui fournit un support à la communication) mais quand elle conduit à figer le contenu ou la forme de l'opération, elle peut aussi constituer un obstacle aux innovations ultérieures, c'est-à-dire aux apprentissages. De plus, le volontarisme d'actions ramassées dans le temps propice à une bonne communication n'est pas toujours compatible avec le temps long de l'apprentissage par essais et erreurs.

Quels sont les facteurs les plus propices à faire de la construction d'équipements publics un enjeux de communication? L'exemple des collèges montre que la taille importante de la collectivité territoriale joue sans doute un rôle : elle lui permet de conduire un volume d'opérations qui contribue à leur visibilité et de se doter des compétences nécessaires pour mener une politique originale. Mais elle ne suffit pas. L'émulation entre collectivités locales concurrentes dans une même région, les références que constituent des opérations antérieures, l'expérience des individus qui à un moment donné sont en mesure d'influer sur la politique, peuvent aussi aider à comprendre que des collectivités territoriales de même taille s'engagent sur des voies très différentes. Notre étude montre ainsi qu'il n'y a sans doute pas de profil type des collectivités territoriales en pointe en matière d'usage de l'architecture à des fins de communication, mais plutôt des circonstances objectives plus ou moins favorables à cet usage, dont il faut de plus que quelqu'un se saisisse. 